\title{
Comprehensive analysis of the characteristics and treatment outcomes of patients with non-small cell lung cancer treated with anti-PD-1 therapy in real-world practice
}

\author{
Beung-Chul Ahn ${ }^{1} \cdot$ Kyoung-Ho Pyo ${ }^{1,2}$. Chun-Feng Xin ${ }^{2}$. Dongmin Jung ${ }^{2} \cdot$ Hyo Sup Shim ${ }^{3}$. Chang Young Lee ${ }^{4}$. \\ Seong Yong Park ${ }^{4} \cdot$ Hong In Yoon ${ }^{5} \cdot$ Min Hee Hong ${ }^{1} \cdot$ Byoung Chul Cho ${ }^{1} \cdot$ Hye Ryun Kim ${ }^{1}$
}

Received: 25 December 2018 / Accepted: 18 March 2019 / Published online: 25 March 2019

(c) The Author(s) 2019

\begin{abstract}
Purpose Immune checkpoint inhibitors (ICI) have shown marked responses in patients with non-small cell lung cancer (NSCLC) in clinical trials. However, because such trials comprise cohorts selected based on specific criteria, it is unclear if their results represent routine clinical practice.

Methods We examined 155 patients with advanced NSCLC who were administered either nivolumab or pembrolizumab at Yonsei Cancer Center, Korea between March 2014 and January 2019. Patient characteristics, EGFR/ALK mutation status, metastatic locations, response to ICIs, and adverse events were retrospectively analyzed.

Results The median age was 64 years and $72.9 \%$ of patients were male; former or current smokers constituted $67.1 \%$ of the subjects. Adenocarcinoma was predominant (67.7\%), and $50.3 \%$ of the patients underwent $\geq 2$ previous treatments. Twentythree patients (14.8\%) were EGFR mutation- or $A L K$ rearrangement-positive. The objective response rate (ORR) was $23.9 \%$ [95\% confidence interval (CI) 17.4-31.4\%]; the median progression-free survival (PFS) and overall survival (OS) were 3.06 (95\% CI 1.893-4.21) and 10.25 (95\% CI 5.39-15.11) months, respectively. Multivariate analysis identified ECOG performance status, EGFR mutation/ALK rearrangement status, liver metastasis and PD-L1 proportion as independent predictors of OS. Furthermore, $61.9 \%$ of the patients had adverse events of any grade; $38.1 \%$ had immune-related adverse events that were associated with PFS and OS on multivariate analysis.

Conclusions The real-world ORR, PFS, OS, and adverse event profiles were comparable to previous clinical trials despite the patients' different baseline characteristics. Our findings can aid in establishing effective immunotherapeutic management of NSCLC in routine clinical practice.
\end{abstract}

Keywords Immunotherapy · Non-small cell lung cancer · Real-world setting $\cdot$ PD-1 · Survival

Beung-Chul Ahn and Kyoung-Ho Pyo contributed equally as cofirst authors.

Byoung Chul Cho and Hye Ryun Kim contributed equally as cocorresponding authors.

Electronic supplementary material The online version of this article (https://doi.org/10.1007/s00432-019-02899-y) contains supplementary material, which is available to authorized users.

Byoung Chul Cho

cbc1971@yuhs.ac

$\triangle$ Hye Ryun Kim nobelg@yuhs.ac

Extended author information available on the last page of the article

\section{Introduction}

Lung cancer is a major cause of cancer-related deaths worldwide (Siegel et al. 2017). Non-small cell lung cancer (NSCLC) accounts for approximately $85 \%$ of all lung cancers, of which approximately $70 \%$ have non-squamous histologies (Herbst et al. 2008; Travis et al. 1995). In 2016, a total of 24,267 new cases of lung cancer were reported in Korea and 17,399 individuals died of this disease, making it the leading cause of cancer-related death in both sexes (Mortality table of Korea 2015).

Patients with advanced lung cancer eventually develop chemotherapy-resistant disease after treatment with conventional cytotoxic agents, demonstrating the necessity for devising other treatment options for refractory NSCLC. 
More recently, programmed cell death protein 1 (PD-1)/programmed death-ligand 1 (PD-L1) immune checkpoint inhibitors (ICIs) showed potent activity against metastatic NSCLC in subsets of clinical trials (Borghaei et al. 2015; Brahmer et al. 2015; Herbst et al. 2016). Some randomized phase III trials have reported a statistically significant achievement in overall survival (OS) with ICIs over docetaxel in patients with platinum-refractory NSCLC: the CheckMate 017 and CheckMate 057 trials in patients with squamous and nonsquamous NSCLC, respectively (both tested nivolumab, a monoclonal-antibody of PD-1) (Borghaei et al. 2015; Brahmer et al. 2015); the Keynote 010 phase II/III trial with pembrolizumab, which also interacts to PD-1 (participation was restricted to at least 1\% PD-L1 expression level on tumor cells) (Herbst et al. 2016). To date, the phase III Keynote 024 and Keynote 042 trials reported that pembrolizumab significantly improves the progression-free survival (PFS) and OS over standard first-line platinum-based chemotherapy; these trials comprise patients with at least 50\% PD-L1 and $1 \%$ PD-L1 expression in their tumor cells, respectively (Lopes et al. 2018; Reck et al. 2016). Based on all these trials, PD-1 inhibitors including nivolumab and pembrolizumab are now approved as standard anticancer treatments for patients with advanced NSCLC.

However, clinical trials have strict and complex enrollment criteria (Garcia et al. 2017). The line of therapy for patient eligibility is usually pre-determined in trials comparing the efficacy of novel investigational products to previous chemotherapies. Thus, the outcomes of these trials do not necessarily represent real-world patients. In particular, there is limited evidence regarding the real-world efficacy of immunotherapy and related clinical findings in patients who are unsuitable for clinical trials or else are categorized into specific subgroups, including those with poor performance status (PS), those with EGFR mutations/ALK rearrangements, and those of Asian ethnicity (Borghaei et al. 2015; Brahmer et al. 2015).

Therefore, we conducted this retrospective analysis of 155 unselected patients with advanced NSCLC; to our knowledge, this study is the largest of its kind performed in Korea. We posited that identifying the clinical characteristics that influence the efficacy of immunotherapy in a real-world setting would be beneficial for validating previous observations and devising an effective immunotherapy strategy in routine clinical practice.

\section{Methods}

\section{Patients and samples}

In total, 155 patients with advanced NSCLC who were administered a PD-1 inhibitor (nivolumab or pembrolizumab) at Yonsei Cancer center between March 2014 and January 2019 were enrolled. Clinical data including patient characteristics, driver gene mutation status, metastatic locations, response to immunotherapy, and adverse events were retrospectively collected and analyzed. The patients were treated with nivolumab at a dose of $3.0 \mathrm{mg} /$ $\mathrm{kg}$ body weight every 2 weeks or pembrolizumab at $200 \mathrm{mg}$ fixed dose every 3 weeks (which represent the licensed dose and administration method in Korea). This study was approved by the institutional review board (IRB no. 4-20160678); the requirement for informed consent was waived.

\section{Assessments}

Patients were assessed for treatment response by computed tomography (CT). Chest CT and abdominal CT were taken every two or three cycles during the treatment. Besides regular follow-up, additional images were acquired according to the physician's discretion. The clinical response to anti-PD-1 treatment was evaluated using these images according to the Response Evaluation Criteria in Solid Tumors (RECIST), version 1.1 (Eisenhauer et al. 2009). The tumor responses to anti-PD-1 treatment were defined as follows: complete response (CR; the disappearance of all target lesions), partial response (PR; $30.0 \%$ or more reduction in the sum of the diameters of the target lesions), progressive disease (PD; $20.0 \%$ or more increase in the sum of the diameters of the target lesions), and stable disease (SD; not in category to qualify as PR or PD). To consider the difference between RECIST criteria and immune modified-RECIST the patients who were assessed as PD had reassessment after 4-8 weeks to confirm it. The objective response rate (ORR) was defined as the proportion of patients with CR or PR, while the disease control rate (DCR) was defined as the proportion of patients with CR, PR, or SD. PFS was defined as the time from the start of anti-PD-1 treatment to disease progression or death. OS was defined as time from the start of anti-PD-1 treatment to death by any cause. Adverse events related to anti-PD-1 treatment were stated according to the Common Terminology Criteria for Adverse Events, version 4.0 [Common Terminology Criteria for Adverse Events (CTCAE) v4.0 (2018)].

\section{Statistical analysis}

The Kaplan-Meier method was used to estimate OS and PFS; subgroups were compared using the log-rank test for total number of patients. Additional propensity score matching analysis for each clinical characteristic were done to reduce the bias due to confounding variables. Univariable and multivariable Cox proportional hazard regression models were adopted to determine hazard ratios with $95 \%$ confidence intervals (CIs). Multivariate analysis was performed 
with adjustment for age, sex, smoking status, number of prior treatment lines, mutational status, brain metastasis, liver metastasis, and PD-L1 expression level. Additionally, due to the time-dependent nature of immune-related adverse events (irAEs), we performed 6-week landmark analyses including patients who achieved disease control (for PFS; $n=111$ ) and those who were alive (for OS; $n=133$ ) at 6 weeks to determine the association between irAEs and survival outcomes. Statistical analyses were performed using SPSS version 23 (IBM Software, Armonk, NY, USA) and GraphPad Prism version 5.00 (GraphPad Software, San Diego, CA, USA).

\section{Identification of PD-L1 expression}

In most cases, tumor PD-L1 expression was determined immunohistochemically using the PD-L1 22C3 pharmDx antibody (Dako North America Inc., Carpinteria, CA, USA) or Ventana PD-L1 SP263 antibody (Ventana Medical Systems, Tucson, AZ, USA) as companion diagnosis. PD-L1 expression levels in tumor cells were determined by the percentage of stained tumor cells in each section, which was estimated in increments of 5\% except for a $1 \%$ value. Patients with at least $1 \%$ of the tumor cells who were stained for PD-L1 were considered positive.

\section{Results}

\section{Clinicopathologic characteristics of the study population}

In total, 155 patients with advanced NSCLC were enrolled (Table 1); the majorities were male (72.9) and aged $\geq 60$ years $(68.4 \%)$. Most patients had adenocarcinoma $(67.7 \%)$ or squamous carcinoma (30.3\%). Twenty-three patients $(14.8 \%)$ had $E G F R$ mutations $(n=22)$ or $A L K$ rearrangement $(n=1)$, and 99 (63.9\%) were identified as PD-L1 positive. Thirty-four patients $(21.9 \%)$ had an ECOG performance status score of 2 or higher at the beginning of the treatment. As opposed to clinical trials, the lines of administered therapies were diverse, as $16(10.3 \%), 61$ (39.4\%), and $78(50.3 \%)$ of the patients received anti-PD- 1 treatment as first-line, second-line, or subsequent to second-line therapy, respectively. At presentation immediately before immunotherapy, the most frequent site of metastasis was the ipsilateral or contralateral lung $(71.0 \%)$, followed by the brain (39.4\%), bone (32.9\%), and adrenal gland (18.1\%). By the time of data lock (January 30, 2019), 49 patients (31.6\%) were alive, $92(59.4 \%)$ had died, and $14(9.0 \%)$ was lost to follow-up. The median follow-up duration for the patients was 17.0 months.
Table 1 Baseline characteristics

\begin{tabular}{|c|c|}
\hline & $n(\%)$ \\
\hline \multicolumn{2}{|l|}{ Age (years) } \\
\hline Median (range) & $64(35-85)$ \\
\hline$<60$ & 49 (31.6) \\
\hline \multicolumn{2}{|l|}{ Sex } \\
\hline Male & $113(72.9)$ \\
\hline Female & $42(27.1)$ \\
\hline \multicolumn{2}{|l|}{ Histology } \\
\hline Adenocarcinoma & $105(67.7)$ \\
\hline Squamous & 47 (30.3) \\
\hline Pleomorphic & $2(1.3)$ \\
\hline Unknown & $1(0.6)$ \\
\hline \multicolumn{2}{|l|}{ Smoking } \\
\hline Never & $51(32.9)$ \\
\hline Former smoker & $61(39.4)$ \\
\hline Current smoker & $43(27.7)$ \\
\hline \multicolumn{2}{|l|}{$E G F R$ and $A L K$} \\
\hline Wild-type (both) & $131(84.5)$ \\
\hline Mutant & $\begin{array}{c}23(14.8) \\
(A L K: n=1)\end{array}$ \\
\hline Unknown & $1(0.7)$ \\
\hline \multicolumn{2}{|l|}{ ECOG PS score } \\
\hline 0 & $23(14.8)$ \\
\hline 1 & $98(63.2)$ \\
\hline 2 & $20(12.9)$ \\
\hline 3 & $14(9.0)$ \\
\hline \multicolumn{2}{|l|}{ Prior treatment lines } \\
\hline 0 & $16(10.3)$ \\
\hline 1 & $61(39.4)$ \\
\hline 2 & $32(20.6)$ \\
\hline 3 & $28(18.1)$ \\
\hline 4 & $8(5.2)$ \\
\hline$\geq 5$ & $10(6.4)$ \\
\hline \multicolumn{2}{|l|}{ Metastasis present } \\
\hline Lung ipsilateral & $95(61.3)$ \\
\hline Lung contralateral & $79(51.0)$ \\
\hline Brain & $61(39.4)$ \\
\hline Bone & $51(32.9)$ \\
\hline Adrenal gland & $28(18.1)$ \\
\hline Liver & $24(15.5)$ \\
\hline
\end{tabular}

ECOG PS Eastern Cooperative Oncology Group performance status, $E G F R$ epidermal growth factor receptor, $A L K$ anaplastic lymphoma kinase

\section{Treatment outcomes and potential predictors}

The treatment outcomes for all patients are shown in S1 Table. The ORR was $23.9 \%(n=37)$, all based on achieving PR. Moreover, 35.5\% of the patients had SD, 29.7\% had PD, and $11.0 \%$ were not evaluated. The median OS 
and PFS were 10.25 months (95\% CI 5.39-15.11) and 3.06 months (95\% CI 1.89-4.21), respectively, for all patients (Fig. 1).

We further investigated patients stratified by clinicopathologic factors. ORRs of patients with PS scores of 0-1 and 2-4 were $29.8 \%$ and $2.9 \%$, respectively; the DCRs of these subgroups were $71.9 \%$ and $14.7 \%$, respectively. The ORRs of patients who were never-smokers, were $E G F R$ mutation/ALK-rearrangement-positive, and had $\geq 50 \%$ PD-L1 expression were $13.7 \%, 13.0 \%$, and $37.7 \%$, respectively; the corresponding DCRs were $51.0 \%$ and $34.7 \%$, and $75.4 \%$, respectively.

We next performed PFS and OS analyses of patients stratified by the above factors and metastatic lesions. Age, sex, and smoking status did not significantly influence OS and PFS. However, median PFS rates of patients with $E G F R$ mutation/ALK rearrangement were significantly shorter than those of wild-type patients (1.6 vs. 3.8 months; $P<0.01)$ as were OS rates (4.4 vs. 13.5 months; $P<0.01)$. Furthermore, the median PFS of patients with $50 \%$ or more PD-L1-positive cells was 6.0 months, which was significantly longer than those with $0-49 \%$ PDL1-positive cells ( 2.9 months; $P<0.01)$; the same was true for OS (20.5 vs. 7.8 months, $P=0.021$ ). Other factors that showed significant differences were PS score, the presence of metastatic lesions (i.e., in the brain and liver), and line of therapy. Kaplan-Meier plots are shown in Fig. 2 and S1 Fig.

Multivariate analysis identified poor PS, EGFR mutation/ALK rearrangement positivity, liver metastasis and low PD-L1 expression as independent negative predictors of OS (Table 2).

a

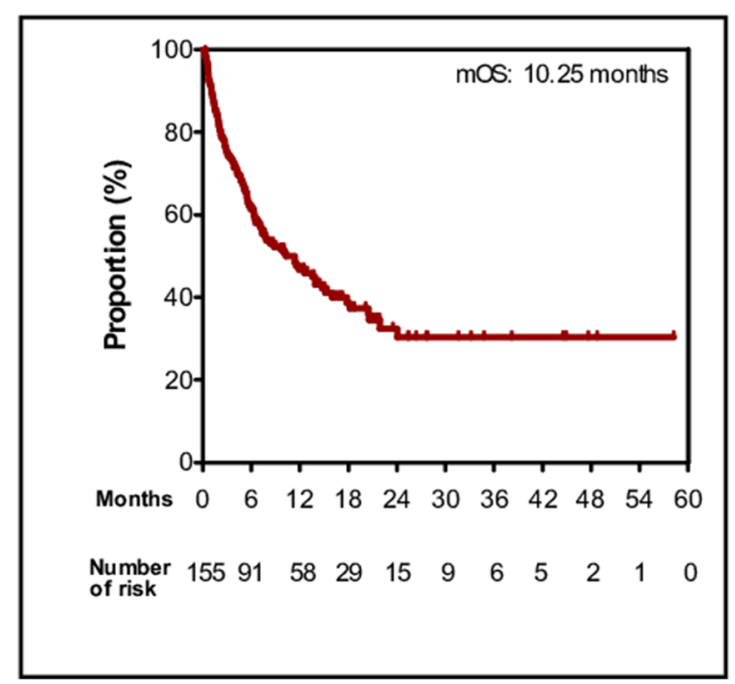

\section{Brain and liver metastasis response}

We also analyzed the treatment outcomes of patients according to brain and/or liver metastasis status. Sixty-one patients had brain metastasis upon commencing ICI therapy, 41 of whom underwent local radiotherapy before or during treatment. Response in the brain was evaluated via magnetic resonance imaging during the same global response evaluation cycle. The patients' intracranial ORR and DCR were $16.4 \%$ (95\% CI 8.16-28.10\%) and 42.6\% (95\% CI 30.02-55.92\%), respectively. The ORR did not differ significantly from the global response rate $(16.4 \%$ vs. $23.9 \%, P=0.23)$, but the DCRs were significantly different $(42.6 \%$ vs. $59.4 \%$, $P=0.026$ ). Furthermore, 25 patients had liver metastasis; their ORR and DCR were $12.0 \%$ (95\% CI 2.55-31.22\%) and $32.0 \%$ (95\% CI $14.95-53.50 \%$ ), respectively, and only the DCR differed significantly from the global response (ORR, $P=0.19$; DCR, $P=0.011)$. The treatment responses of each region for patients with brain and liver metastases are shown in S2 Table.

\section{Adverse events and their association with clinical outcomes}

The proportion of patients who experienced adverse events of any type and grade was $61.9 \%$. The three most common adverse events were rash (19.4\%), decreased appetite (12.3\%), and fatigue (11.6\%); none were grade $\geq 3$. The most common grade $\geq 3$ adverse event was pneumonia $(6.5 \%)$ followed by pneumonitis (3.2\%).

Based on the previous studies, we defined irAEs as adverse events with a potential immunological basis that require

\section{b Total Progression Free Suvival}

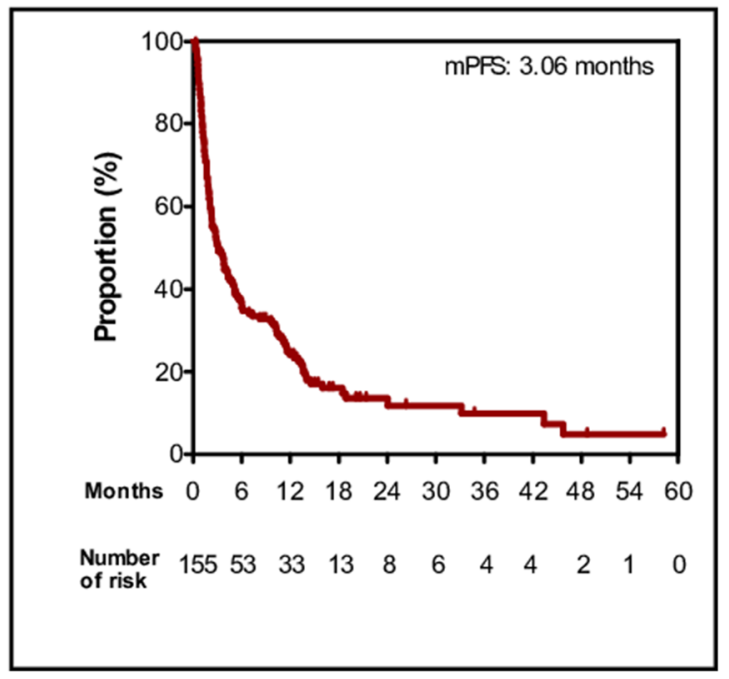

Fig. 1 Kaplan-Meier plot for the total population $(n=139)$. a Overall survival (OS) and b progression-free survival (PFS) from the beginning of anti-PD-1 treatment. $P D-L 1$ programmed death-ligand 1 
immunosuppressive or endocrine therapy (Friedman et al. 2016). We strictly considered only those irAEs that medical professionals could recognize objectively via physical examination or laboratory results; this also helped to reduce bias. IrAEs observed in our patients are shown in Table 3. The pneumonitis was distinguished from pneumonia by sputum culture plus laboratory C-reactive protein (CRP) and procalcitonin level. Most of patients who were diagnosed as pneumonitis had process of multidisciplinary team meeting and/or consultation to pulmonologists (S3 Table).

We categorized hypothyroidism, adrenal insufficiency, and panhypopituitarism as endocrine irAEs for further analysis. The proportion of patients who experienced irAEs was $38.1 \%$ and $5.16 \%$ experienced grade $\geq 3$ irAEs. Sixweek landmark analysis showed that the ORR of patients with irAEs was higher than in those without, although not significantly different [ 21 of 51 patients $(41.2 \%)$ vs. 16 of 60 patients $(26.7 \%) ; P=0.11$ ]. However, the development of irAEs was significantly associated with longer PFS [11.63 months (95\% CI 9.21-14.05) vs. 3.27 months (95\% CI 2.01-4.17); $P<0.001]$ and OS [24.05 months (95\% CI NR-NR) vs. 7.39 months (95\% CI 3.49-11.29); $P<0.001]$. Upon further analysis of irAE subtypes, patients with skin rash had significantly longer PFS [11.40 months (95\% CI 7.87-14.93) vs. 5.09 months (95\% CI 3.53-6.66); $P=0.008]$ and OS [NR (95\% CI NR-NR) vs. 11.37 months (95\% CI 5.84-16.89); $P=0.004]$. Patients with endocrine irAEs had longer PFS [10.22 months (95\% CI 6.04-14.39) vs. 5.09 months (95\% CI 3.49-6.70); $P=0.054]$ and OS [NR (95\% CI NR-NR) vs. 12.58 months (95\% CI 8.02-17.15); $P=0.037]$, although the differences were not significant for PFS (Fig. 3).

Multivariate analysis revealed that the presence of any irAEs was significantly associated with increased PFS and OS based on the 6-week landmark analysis, whereas skin irAEs and endocrine irAEs subsets were not. Overall, skin and endocrine irAEs were not identified as significant positive predictive factors, though they showed a tendency as such (Table 4).

Additional 12-week and 24-week landmark analysis were also done and are shown on online resource (S4 Table and S2 Fig.) The result of these analyses showed similar trend as 6-week landmark but 12-week analysis only showed significance between the presence of any irAEs and OS not with PFS. 24-week analysis did not show significance and only showed similar trends.

\section{Discussion}

Currently, the National Clinical Cancer Network and American Society of Clinical Oncology guidelines do not recommend more than two consecutive lines of cytotoxic chemotherapy for advanced NSCLC; intervening or subsequent immunotherapy, best supportive care, or participation in a clinical trial is otherwise recommended (Rizvi et al. 2015; Rozenblum et al. 2017). In contrast to chemotherapy or tyrosine kinase inhibitors, ICIs have the ability to restore a patient's antitumor immunity, allowing the destruction of malignant cells with the potential for a robust and durable clinical response (Wang et al. 2014). In the CheckMate 017 (squamous NSCLC) and CheckMate 057 (non-squamous NSCLC) clinical trials for nivolumab, the ORRs were $20.0 \%$ and $19.0 \%$, median PFS rates were 3.5 and 2.3 months, and median OS rates were 9.2 and 12.2 months, respectively (Borghaei et al. 2015; Brahmer et al. 2015). The Keynote 001 study of pembrolizumab for patients with advanced NSCLC reported an objective response rate of $19.4 \%$, median PFS of 3.7 months, and median OS of 12 months (Garon et al. 2015). Hence, immunotherapy has become an indispensable option for any line of therapy for advanced NSCLC.

The ORR, PFS, and OS in our study $(23.9 \%, 3.06$ months, and 10.25 months, respectively) were comparable to those of the previous studies. Although we enrolled unselected patients who were ineligible for the previous trials or participated as minor subset cohorts (including those with poor PS, EGFR mutations/ALK rearrangements, and brain and liver metastases, which are factors known to be indicators of unfavorable responses to immunotherapy) (Tamiya et al. 2018), the clinical outcomes were similar to the previously published results. This could be partially explained by higher PD-L1 expression (40.5\% of patients in our study had PD-L1 expression levels $\geq 50 \%$; whereas $23.3 \%$ of patients exhibited PD-L1 levels $\geq 50 \%$ in the Keynote-001 study and 37.2\% exhibited PD-L1 levels $\geq 10 \%$ in the CheckMate-057 study) (Borghaei et al. 2015; Garon et al. 2015). Higher PD-L1 expression is reportedly a favorable predictor of immunotherapy efficacy, and levels of this protein are usually higher among Asians (Lin et al. 2016; Patel and Kurzrock 2015). Furthermore, ethnicity itself could have been a factor, since the abovementioned studies comprised mostly Caucasian patients, whereas ours were Asian.

Our data indicated that harboring EGFR mutations/ALK rearrangements was associated with poorer PFS and OS, which was consistent with recent retrospective and prospective studies (Lee et al. 2017). Preliminary studies suggest that sensitivity to ICIs is high in tumors with high somatic mutations (Rizvi et al. 2015; Rozenblum et al. 2017). Therefore, never-smokers and patients with EGFR mutations/ALK rearrangements are known to have poorer outcomes because of their low mutational burdens, although our study did not reveal an association between smoking status and survival outcomes. Subgroup analyses of both the CheckMate 057 (Borghaei et al. 2015) and Keynote 010 (Herbst et al. 2016) prospective trials revealed no significant OS differences 
a

Overall suvival

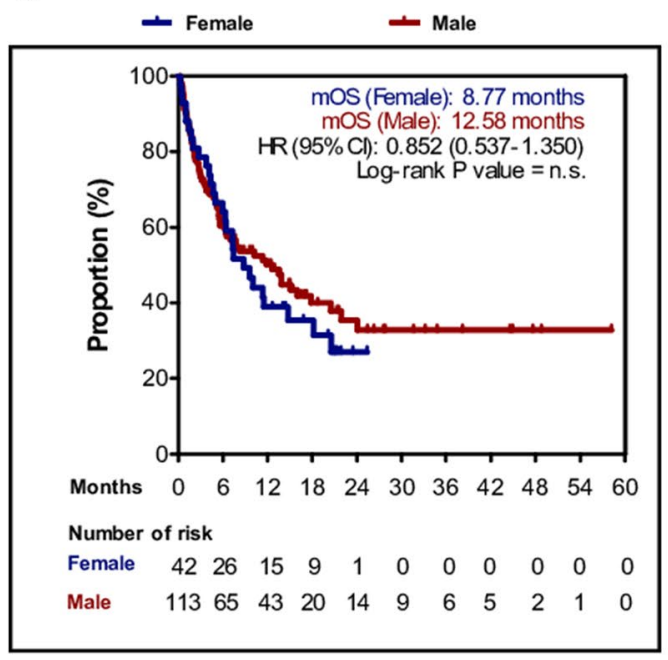

C

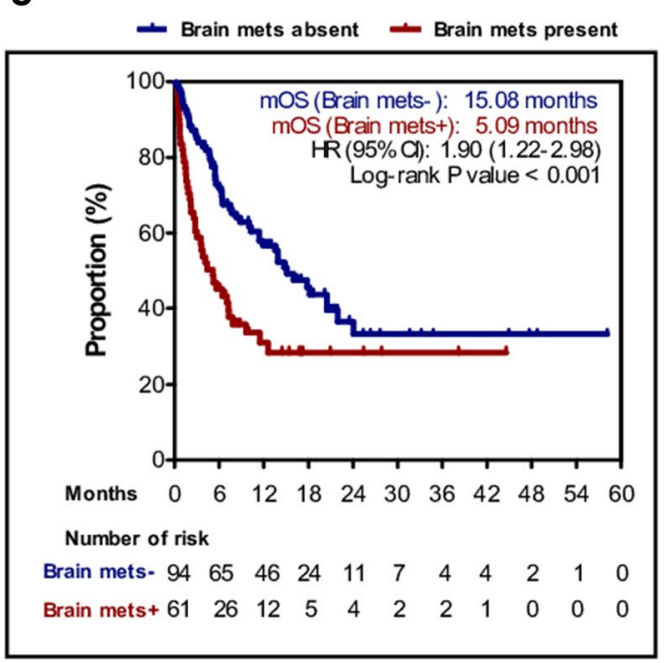

e

Overall suvival

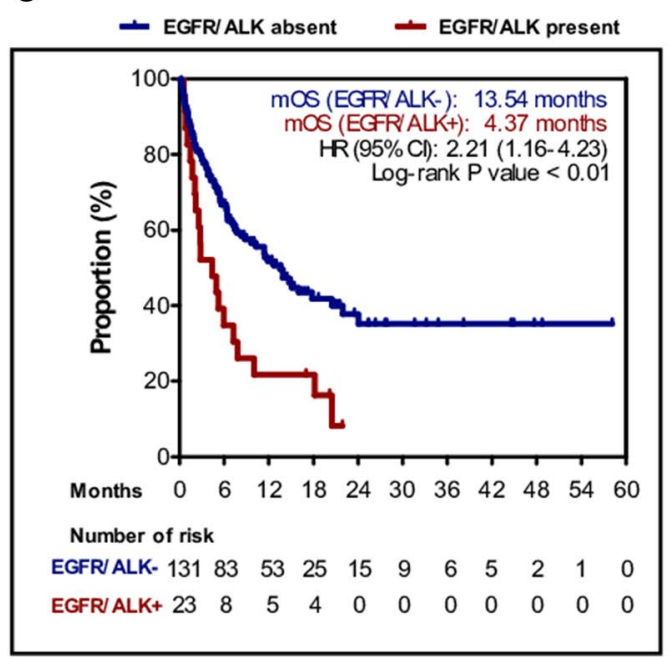

b

Overall suvival

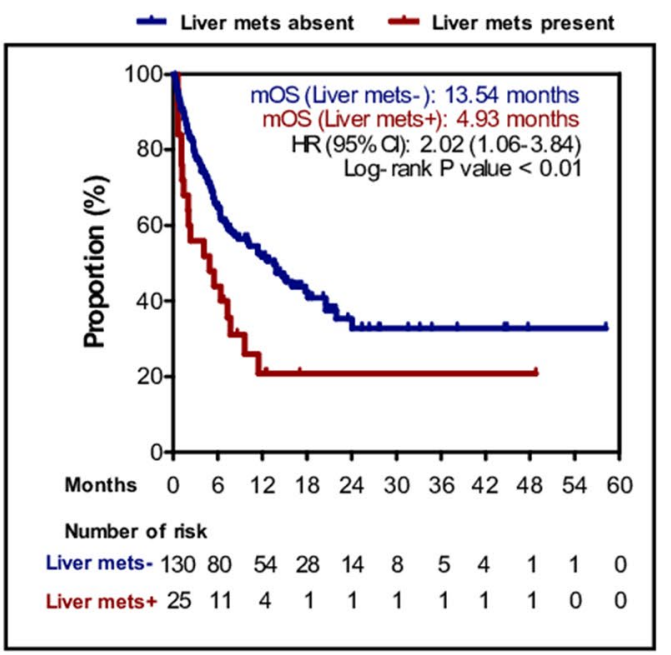

d

\section{Overall suvival}

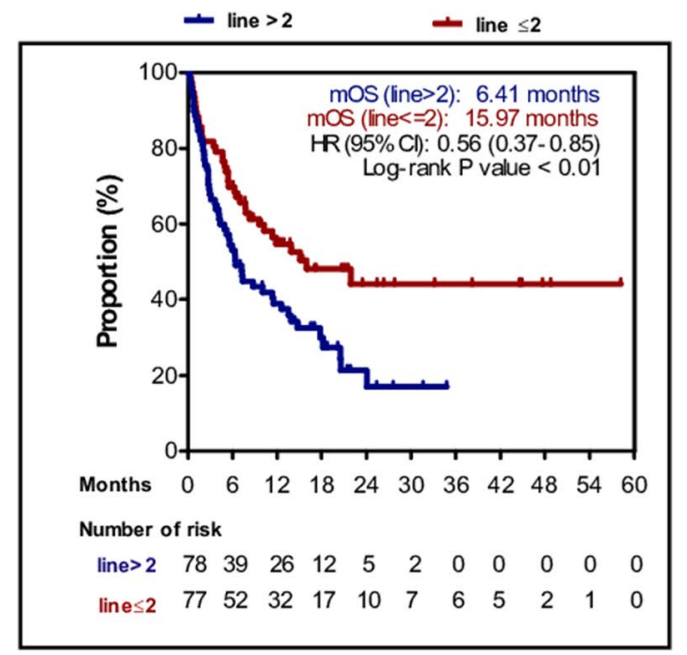

\section{f Overall suvival}

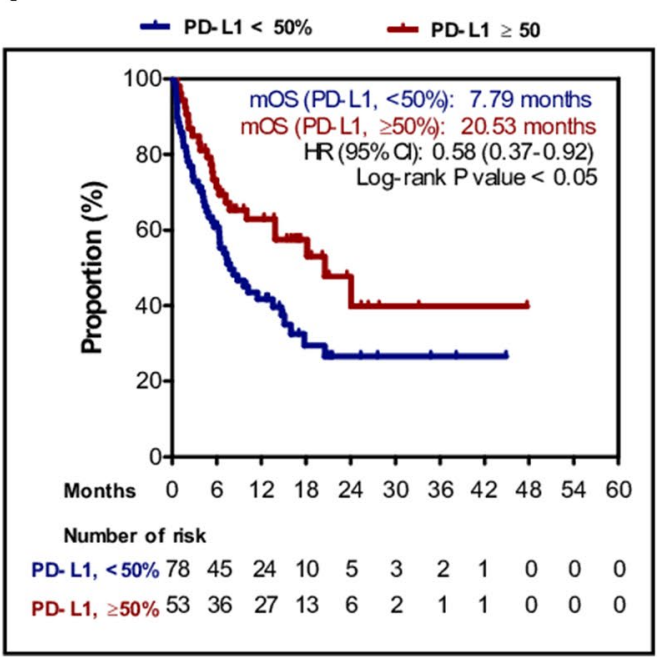


४Fig. 2 Kaplan-Meier plot for the overall survival (OS) stratified by clinical factors. a Sex; b liver metastasis; c brain metastasis; $\mathbf{d}$ line of therapy; e EGFR/ALK mutation status; f PD-L1 expression level of 50\%. $P D-L 1$ programmed death-ligand 1, EGFR epidermal growth factor receptor, $A L K$ anaplastic lymphoma kinase, $H R$ hazard ratio, n.s. not significant, $U D$ undetermined

based on $E G F R$ mutation status. Overall, excluding patients with $E G F R$ mutations/ALK rearrangements ought to produce a higher response rate to anti-PD-1 immunotherapy in routine clinical practice.

Our results also suggested that PD-L1 expression levels $\geq 50 \%$ were well-correlated with improved PFS and OS rates following anti-PD-1 treatment. As PD-L1 expression alone is accepted as an imperfect biomarker for predicting prognosis, it remains debated whether PD-L1 expression levels should be used as a benchmark for prescribing ICIs (Herbst et al. 2014; Patel and Kurzrock 2015). However, our results imply that determining PD-L1 status can help identify patients most likely to benefit from anti-PD-1 treatment in real-world settings.

The existence of liver or brain metastases at the commencement of immunotherapy was associated with poor PFS and OS in our study. Patients with advanced NSCLC who have liver or brain metastases and are receiving chemotherapy or tyrosine kinase inhibitors are known to have poorer prognoses than those with metastases in other locations (Hoang et al. 2012). There are only few retrospective studies regarding immunotherapy outcomes and their association with metastatic lesions. One such retrospective study showed a relationship between the metastatic site and PFS in patients with advanced NSCLC who were treated with nivolumab (Tamiya et al. 2018), while another found that nivolumab was effective against brain metastasis (Gauvain et al. 2018). In our study, we revealed not only differences in ORR and PFS, but also in OS in patients who received anti-PD-1. This supports considering the metastatic lesion site a criterion for selecting candidates for immunotherapy.

Finally, our data indicated that the development of certain irAEs is associated with improved anti-PD-1 treatment efficacy in patients with NSCLC, which is consistent with earlier studies (Suresh et al. 2018; Teraoka et al. 2017). A previous retrospective study showed that thyroid dysfunction irAE is associated with a better prognosis in patients with NSCLC (Osorio et al. 2017). Several other retrospective studies have demonstrated similar associations in patients with dermatological irAEs who were treated for melanoma and NSCLC with ICIs (FreemanKeller et al. 2016; Hasan Ali et al. 2016). Most recently, Haratani et al. performed a landmark analysis that revealed significant differences in both PFS and OS between NSCLC patients with vs. without irAEs [hazard ratios of 0.525 (95\% CI $0.287-0.937$ ); $P=0.03$ for PFS and 0.282 (95\% CI $0.101-0.667) ; P=0.003$ for OS on multivariate analysis] (Haratani et al. 2018; Teraoka et al. 2017) Our own 6-week landmark analysis revealed significant differences in OS and PFS when patients were stratified by existence of irAEs, also multivariate analysis confirmed a significant difference for PFS and OS. Our study included a greater number of covariables than those examined by Haratan et al.; we additionally investigated PD-L1 status, PS, and liver metastasis (Table 4). Our comprehensive landmark analyses suggest that the early onset of irAEs is predictive of response or of the durable clinical benefits in patients with NSCLC treated with PD-1 inhibitors, thereby possibly aiding clinicians in improving immunotherapy planning, including whether to switch or cease treatment, during the interval before the routine response evaluation.

To the best of our knowledge, ours is one of the largest comprehensive retrospective studies of real-world patients who were treated with PD-1 inhibitors. Previous retrospective studies examined real-world situations only partially, and their findings were, therefore, confined in scope. For example, some studies included only patients who were previously treated, while others only considered patients who received a single immunotherapy agent. Furthermore, due to insufficient follow-up periods, most of the previous retrospective studies were unable to determine matured OS dates (Fujimoto et al. 2018; Garassino et al. 2018; Kobayashi et al. 2018). In contrast, our study comprised near-complete results for both efficacy and safety; in addition, it compared the outcomes of patients with specific clinical factors and adverse events in detail.

In conclusion, our study provided comprehensive clinical characteristics and outcomes of patients with NSCLC who received anti-PD-1 treatment in Korea. In the context of heterogeneous real-world settings, further efforts are required to develop efficient therapeutic strategies, ranging from proper patient selection to determining the correct timing of administering immunotherapy. 
Table 2 Cox proportional hazards regression analysis of the effects of clinical factors on overall survival
Table 3 Treatment-related adverse events according to category and grade

\begin{tabular}{|c|c|c|c|c|c|c|}
\hline \multirow[t]{2}{*}{ Category } & \multicolumn{3}{|c|}{ Univariate } & \multicolumn{3}{|c|}{ Multivariate } \\
\hline & HR & $95 \%$ CI & $P$ value & HR & $95 \%$ CI & $P$ value \\
\hline Age (<75 vs. $\geq 75$ years $)$ & 1.049 & $0.593-1.854$ & 0.871 & 0.712 & $0.337-1.502$ & 0.372 \\
\hline Sex (male vs. female) & 1.167 & $0.748-1.822$ & 0.496 & 0.527 & $0.150-1.848$ & 0.317 \\
\hline ECOG PS (0- 1 vs. $2-3)$ & 6.989 & $4.391-11.12$ & $<0.001$ & 7.566 & $4.008-14.282$ & $<0.001$ \\
\hline Smoking (never vs. current or former) & 1.022 & $0.645-1.620$ & 0.925 & 0.878 & $0.258-2.987$ & 0.835 \\
\hline PD-L1 (<50\% vs. $\geq 50 \%)$ & 0.631 & $0.398-0.999$ & 0.049 & 0.430 & $0.250-0.741$ & 0.002 \\
\hline Liver metastases (absent vs. present) & 2.045 & $1.228-3.406$ & 0.006 & 2.388 & $1.263-4.513$ & 0.007 \\
\hline Brain metastases (absent vs. present) & 1.926 & $1.272-2.917$ & 0.002 & 1.601 & $0.935-2.741$ & 0.086 \\
\hline Prior treatment line ( $\geq 2$ vs. $<2$ ) & 1.777 & $1.170-2.700$ & 0.007 & 1.202 & $0.685-2.109$ & 0.521 \\
\hline $\begin{array}{l}E G F R \text { mutation or } A L K \text { rearrangement } \\
\text { (absent vs. present) }\end{array}$ & 2.230 & $1.352-3.676$ & 0.002 & 2.711 & $1.377-5.338$ & 0.0024 \\
\hline
\end{tabular}

$H R$ hazard ratio, CI confidence interval, ECOG PS Eastern Cooperative Oncology Group performance status (score), $P D-L 1$ programmed death-ligand 1

\begin{tabular}{lllll}
\hline Adverse events & $\begin{array}{l}\text { No. of subjects (all } \\
\text { grades) }\end{array}$ & $\begin{array}{l}\text { Percentage (all } \\
\text { grades) }\end{array}$ & $\begin{array}{l}\text { No. of subjects } \\
\text { (grades 3-4) }\end{array}$ & $\begin{array}{l}\text { Percentage } \\
\text { (grades 3-4) }\end{array}$ \\
\hline Any AEs & & & 0 & 0 \\
Decrease appetite & 19 & 12.3 & 0 & 0 \\
Fatigue & 18 & 11.6 & 1 & 0.7 \\
Dyspnea & 16 & 10.3 & 10 & 6.5 \\
Pneumonia & 15 & 10.3 & 0 & 0 \\
Nausea/vomiting & 4 & 2.6 & 0 & 0 \\
Pyrexia & 3 & 1.9 & 0 & 0 \\
Constipation & 3 & 1.9 & 0 & 0 \\
Edema & 2 & 1.3 & 0 & 0 \\
Neuropathy & 1 & 0.7 & 0 & 0 \\
Infusion reaction & 1 & 0.7 & & 0 \\
Immune-related AEs & & & 0 & 3.2 \\
Rash & 30 & 19.4 & 5 & 0.7 \\
Pneumonitis & 11 & 12.2 & 1 & 0 \\
Diarrhea & 10 & 6.5 & 0 & 0 \\
Hypothyroidism & 10 & 6.5 & 0 & 0.7 \\
AST/ALT elevation & 5 & 3.2 & 1 & 0 \\
Hemolytic anemia & 3 & 1.9 & 1 & 0.7 \\
Adrenal insufficiency & 3 & 0.7 & 0 & \\
Panhypopituitarism & 1 & & 0.9 & 0 \\
\hline AFadversevent AlT & & & 0.7 & \\
\hline
\end{tabular}

$A E$ adverse event, $A L T$ alanine aminotransferase, $A S T$ aspartate aminotransferase 


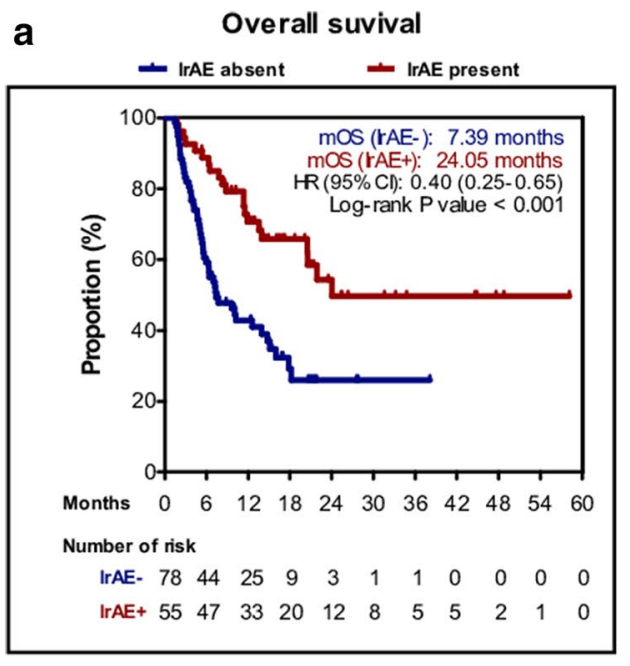

c

Overall suvival

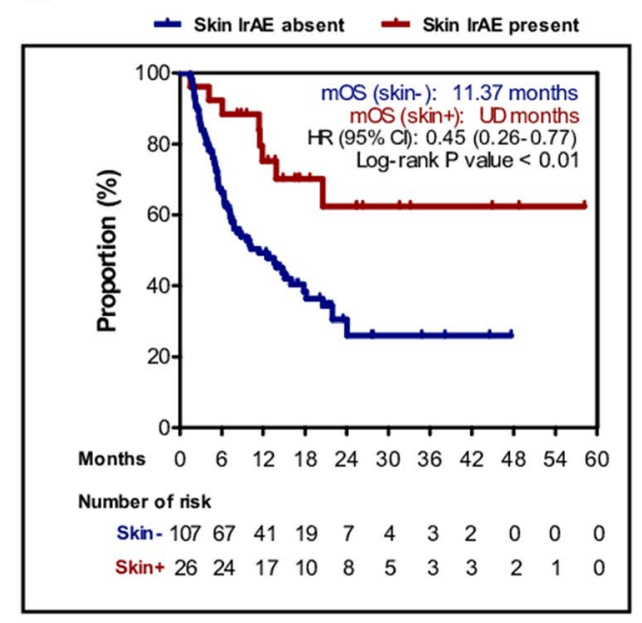

e

Overall suvival

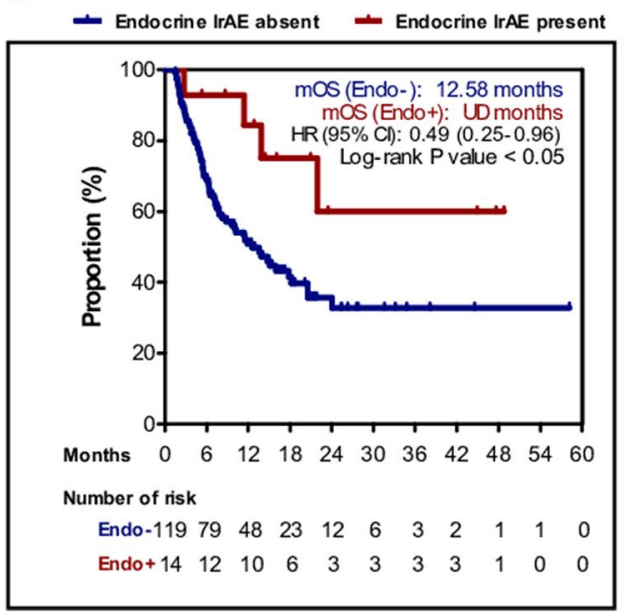

Fig. 3 Kaplan-Meier plot with 6-week landmark analysis for the overall survival (OS) and progression-free survival (PFS) stratified by the presence of irAEs. By any irAEs a OS, b PFS; by skin irAEs c

\section{b Progression free suvival}

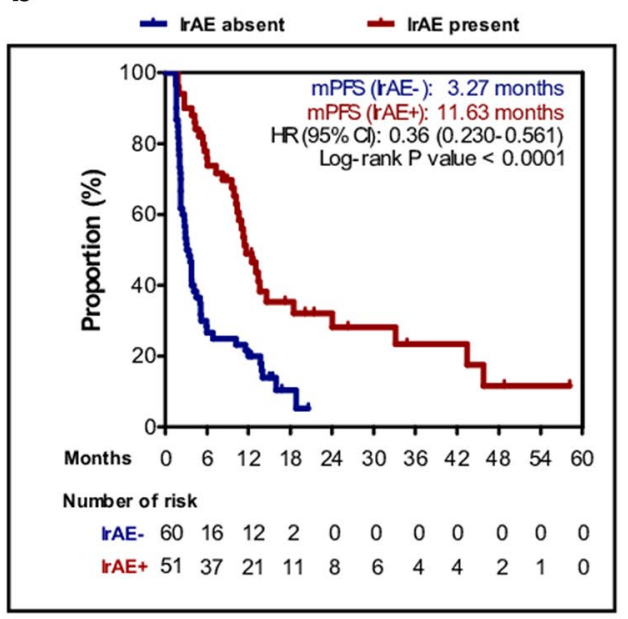

d Progression free suvival

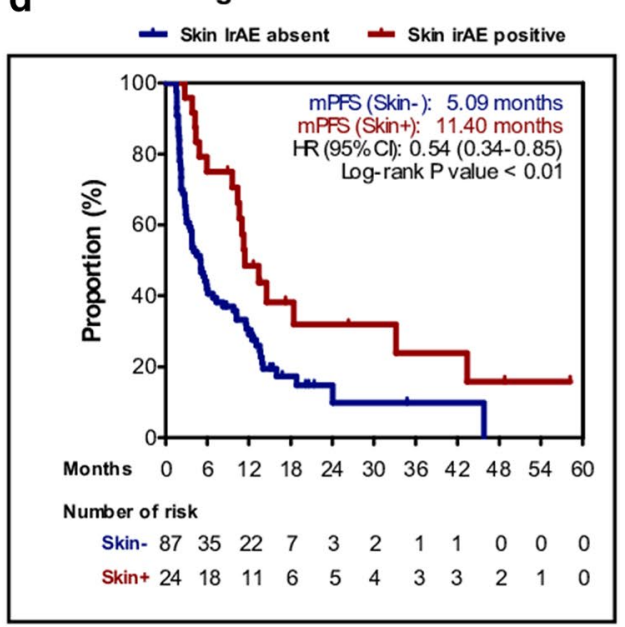

f Progression free suvival

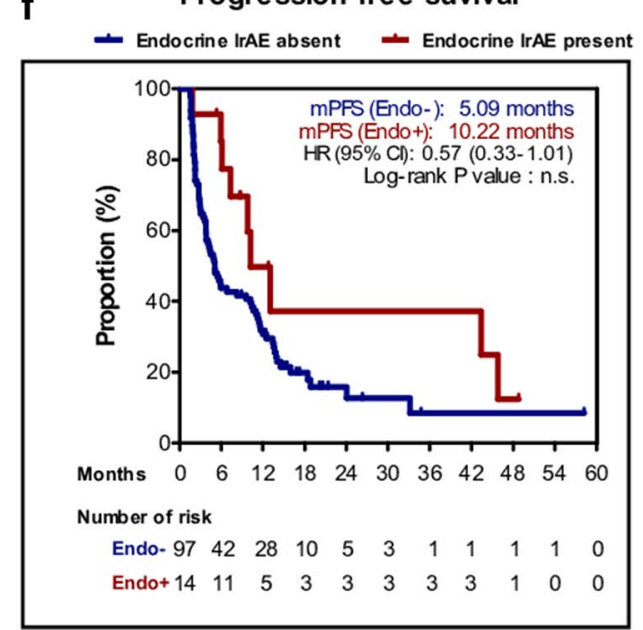

OS, d PFS; by endocrine irAEs e OS, f PFS. irAE immune-related adverse event, $H R$ hazard ratio, n.s. not significant, $U D$ undetermined 
Table 4 Cox proportional hazards regression analysis of the effect of irAE development on PFS and OS (6-week landmark)

\begin{tabular}{|c|c|c|c|c|c|c|}
\hline \multirow[t]{2}{*}{ Survival } & \multicolumn{3}{|c|}{ Univariate } & \multicolumn{3}{|c|}{ Multivariate } \\
\hline & HR & $95 \% \mathrm{CI}$ & $P$ value & HR & $95 \% \mathrm{CI}$ & $P$ value \\
\hline \multicolumn{7}{|c|}{ PFS (6-week landmark), $N=111$} \\
\hline Any irAEs & 0.367 & $0.233-0.579$ & $<0.001$ & 0.434 & $0.256-0.735$ & 0.002 \\
\hline Skin irAEs & 0.488 & $0.284-0.837$ & 0.009 & 0.643 & $0.350-1.180$ & 0.154 \\
\hline Endocrine irAEs & 0.503 & $0.246-1.027$ & 0.059 & 0.368 & $0.132-1.028$ & 0.057 \\
\hline Pneumonitis irAE & 0.685 & $0.276-1.702$ & 0.416 & 1.686 & $0.618-4.597$ & 0.307 \\
\hline \multicolumn{7}{|c|}{ OS (6-week landmark), $N=133$} \\
\hline Any irAEs & 0.383 & $0.228-0.644$ & $<0.001$ & 0.484 & $0.255-0.919$ & 0.027 \\
\hline Skin irAEs & 0.350 & $0.167-0.734$ & 0.005 & 0.420 & $0.162-1.087$ & 0.074 \\
\hline Endocrine irAEs & 0.358 & $0.130-0.983$ & 0.046 & 0.255 & $0.051-1.288$ & 0.098 \\
\hline Pneumonitis irAE & 1.252 & $0.503-3.113$ & 0.629 & 4.117 & $1.420-11.942$ & 0.009 \\
\hline
\end{tabular}

Covariables included age ( $\geq 75$ vs. $<75$ years), sex (male vs. female), Eastern Cooperative Oncology Group performance status score ( $0-1$ vs. $2-3$ ), smoking status (current or former vs. never), PD-L1 ( $<50 \%$ vs $\geq 50 \%$ ), liver metastasis present (yes vs. no), brain metastasis present (yes vs. no), number of prior treatment lines ( $\geq 2$ vs. $<2$ ), and $E G F R$ mutation or $A L K$ rearrangement (absent vs. present)

irAE immune-related adverse events, $E G F R$ epidermal growth factor receptor, $A L K$ anaplastic lymphoma kinase, $P F S$ progression-free survival, $O S$ overall survival, $H R$ hazard ratio, $C I$ confidence interval

\begin{abstract}
Acknowledgements This work was supported by Grants from the Basic Science Research Program through the National Research Foundation (NRF) of Republic of Korea (2017R1D1A1B03029874, 2017M3A9E8029717, 2017M3A9E9072669, 2016M3C9A4922809).
\end{abstract}

\section{Compliance with ethical standards}

Conflict of interest The authors declare no potential conflicts of interest.

Ethical approval All procedures performed in studies involving human participants were in accordance with the ethical standards of the institutional review board of Severance Hospital and with the 1964 Helsinki declaration and its later amendments or comparable ethical standards.

Informed consent Informed consent was waived due to the retrospective nature of this study, approved by the institutional review board (IRB no. 4-2016-0678).

Open Access This article is distributed under the terms of the Creative Commons Attribution 4.0 International License (http://creativeco mmons.org/licenses/by/4.0/), which permits unrestricted use, distribution, and reproduction in any medium, provided you give appropriate credit to the original author(s) and the source, provide a link to the Creative Commons license, and indicate if changes were made.

\section{References}

Borghaei $\mathrm{H}$ et al (2015) Nivolumab versus docetaxel in advanced nonsquamous non-small-cell lung cancer. N Engl J Med 373:16271639. https://doi.org/10.1056/NEJMoa1507643

Brahmer J et al (2015) Nivolumab versus docetaxel in advanced squamous-cell non-small-cell lung cancer. N Engl J Med 373:123-135. https://doi.org/10.1056/NEJMoa1504627

Common Terminology Criteria for Adverse Events (CTCAE) v4.0 (2018) https://ctep.cancer.gov/protocolDevelopment/electronic _applications/ctc.htm. Accessed 31 Jan 2018
Eisenhauer EA et al (2009) New response evaluation criteria in solid tumours: revised RECIST guideline (version 1.1). Eur J Cancer 45:228-247. https://doi.org/10.1016/j.ejca.2008.10.026

Freeman-Keller M, Kim Y, Cronin H, Richards A, Gibney G, Weber JS (2016) Nivolumab in resected and unresectable metastatic melanoma: characteristics of immune-related adverse events and association with outcomes. Clin Cancer Res 22:886-894. https:// doi.org/10.1158/1078-0432.Ccr-15-1136

Friedman CF, Proverbs-Singh TA, Postow MA (2016) Treatment of the immune-related adverse effects of immune checkpoint inhibitors: a review. JAMA Oncol 2:1346-1353. https://doi.org/10.1001/ jamaoncol.2016.1051

Fujimoto D et al (2018) Efficacy and safety of nivolumab in previously treated patients with non-small cell lung cancer: a multicenter retrospective cohort study. Lung Cancer (Amst, Neth) 119:14-20. https://doi.org/10.1016/j.lungcan.2018.02.017

Garassino MC et al (2018) Italian nivolumab expanded access program in nonsquamous non-small cell lung cancer patients: results in never-smokers and EGFR-mutant patients. J Thorac Oncol 13:1146-1155. https://doi.org/10.1016/j.jtho.2018.04.025

Garcia S et al (2017) Thoracic oncology clinical trial eligibility criteria and requirements continue to increase in number and complexity. J Thorac Oncol 12:1489-1495. https://doi.org/10.1016/j. jtho.2017.07.020

Garon EB et al (2015) Pembrolizumab for the treatment of non-smallcell lung cancer. N Engl J Med 372:2018-2028. https://doi. org/10.1056/NEJMoa1501824

Gauvain C et al (2018) Intracerebral efficacy and tolerance of nivolumab in non-small-cell lung cancer patients with brain metastases. Lung Cancer (Amst, Neth) 116:62-66. https://doi. org/10.1016/j.lungcan.2017.12.008

Haratani K et al (2018) Association of immune-related adverse events with nivolumab efficacy in non-small cell lung cancer. JAMA Oncol 4:374-378. https://doi.org/10.1001/jamaoncol.2017.2925

Hasan Ali O et al (2016) Characterization of nivolumab-associated skin reactions in patients with metastatic non-small cell lung cancer. Oncoimmunology 5:e1231292 https://doi.org/10.1080/21624 02X.2016.1231292

Herbst RS, Heymach JV, Lippman SM (2008) Lung cancer. N Engl J Med 359:1367-1380. https://doi.org/10.1056/NEJMra0802714 
Herbst RS et al (2014) Predictive correlates of response to the anti-PDL1 antibody MPDL3280A in cancer patients. Nature 515:563567. https://doi.org/10.1038/nature14011

Herbst RS et al (2016) Pembrolizumab versus docetaxel for previously treated, PD-L1-positive, advanced non-small-cell lung cancer (KEYNOTE-010): a randomised controlled trial. Lancet 387:1540-1550. https://doi.org/10.1016/S0140-6736(15)01281-7

Hoang T, Dahlberg SE, Sandler AB, Brahmer JR, Schiller JH, Johnson DH (2012) Prognostic models to predict survival in non-small-cell lung cancer patients treated with first-line paclitaxel and carboplatin with or without bevacizumab. J Thorac Oncol 7:1361-1368. https://doi.org/10.1097/JTO.0b013e318260e106

Kobayashi K et al (2018) Real-world efficacy and safety of nivolumab for advanced non-small-cell lung cancer: a retrospective multicenter analysis. Clin Lung Cancer 19:e349-e358. https://doi. org/10.1016/j.cllc.2018.01.001

Lee CK, Man J, Lord S, Links M, Gebski V, Mok T, Yang JC (2017) Checkpoint inhibitors in metastatic EGFR-mutated non-small cell lung cancer-a meta-analysis. J Thorac Oncol 12:403-407. https:// doi.org/10.1016/j.jtho.2016.10.007

Lin Z, Xu Y, Zhang Y, He Q, Zhang J, He J, Liang W (2016) The prevalence and clinicopathological features of programmed deathligand 1 (PD-L1) expression: a pooled analysis of literatures. Oncotarget 7:15033-15046. https://doi.org/10.18632/oncotarget .7590

Lopes $\mathrm{G}$ et al (2018) Pembrolizumab (pembro) versus platinum-based chemotherapy (chemo) as first-line therapy for advanced/metastatic NSCLC with a PD-L1 tumor proportion score (TPS) $\geq 1 \%$ : open-label, phase 3 KEYNOTE-042 study. J Clin Oncol 36:abstr LBA4

Mortality table of Korea (2015) http://kosis.kr. Accessed 12 Aug 2018

Osorio JC et al (2017) Antibody-mediated thyroid dysfunction during T-cell checkpoint blockade in patients with non-small-cell lung cancer. Ann Oncol 28:583-589. https://doi.org/10.1093/annonc/ mdw640

Patel SP, Kurzrock R (2015) PD-L1 expression as a predictive biomarker in cancer immunotherapy. Mol Cancer Ther 14:847
Reck M et al (2016) Pembrolizumab versus chemotherapy for PDL1-positive non-small-cell lung cancer. N Engl J Med 375:18231833. https://doi.org/10.1056/NEJMoa1606774

Rizvi NA et al (2015) Cancer immunology. Mutational landscape determines sensitivity to PD-1 blockade in non-small cell lung cancer. Science 348:124-128. https://doi.org/10.1126/science.aaa1348

Rozenblum AB, Ilouze M, Dudnik E, Dvir A, Soussan-Gutman L, Geva S, Peled N (2017) Clinical impact of hybrid capture-based nextgeneration sequencing on changes in treatment decisions in lung cancer. J Thorac Oncol 12:258-268. https://doi.org/10.1016/j. jtho.2016.10.021

Siegel RL, Miller KD, Jemal A (2017) Cancer statistics, 2017. CA Cancer J Clin 67:7-30. https://doi.org/10.3322/caac.21387

Suresh K et al (2018) Pneumonitis in Non-small cell lung cancer patients receiving immune checkpoint immunotherapy: incidence and risk factors. J Thorac Oncol 13:1930-1939. https:// doi.org/10.1016/j.jtho.2018.08.2035

Tamiya M et al (2018) Metastatic site as a predictor of nivolumab efficacy in patients with advanced non-small cell lung cancer: a retrospective multicenter trial. PLoS One 13:e0192227. https:// doi.org/10.1371/journal.pone.0192227

Teraoka S et al (2017) Early immune-related adverse events and association with outcome in advanced non-small cell lung cancer patients treated with nivolumab: a prospective cohort study. J Thorac Oncol 12:1798-1805. https://doi.org/10.1016/j. jtho.2017.08.022

Travis WD, Travis LB, Devesa SS (1995) Lung cancer. Cancer 75:191-202

Wang $C$ et al (2014) In vitro characterization of the anti-PD-1 antibody nivolumab, BMS-936558, and in vivo toxicology in nonhuman primates. Cancer Immunol Res 2:846-856. https://doi. org/10.1158/2326-6066.Cir-14-0040

Publisher's Note Springer Nature remains neutral with regard to jurisdictional claims in published maps and institutional affiliations.

\section{Affiliations}

\section{Beung-Chul Ahn ${ }^{1} \cdot$ Kyoung-Ho Pyo ${ }^{1,2} \cdot$ Chun-Feng Xin ${ }^{2} \cdot$ Dongmin Jung ${ }^{2} \cdot$ Hyo Sup Shim ${ }^{3} \cdot$ Chang Young Lee $^{4}$. Seong Yong Park ${ }^{4}$ Hong In Yoon ${ }^{5}$ Min Hee Hong ${ }^{1} \cdot$ Byoung Chul Cho ${ }^{1} \cdot$ Hye Ryun Kim ${ }^{1}$}

1 Division of Medical Oncology, Department of Internal Medicine, Yonsei Cancer Center, Yonsei University College of Medicine, 50 Yonsei-ro, Seodaemun-gu, Seoul 120-752, Republic of Korea

2 Severance Biomedical Science Institute, Yonsei University College of Medicine, Seoul, Republic of Korea

3 Department of Pathology, Severance Hospital, Yonsei University College of Medicine, Seoul, Republic of Korea
4 Department of Thoracic and Cardiovascular Surgery, Yonsei University College of Medicine, Seoul, Republic of Korea

5 Department of Radiation Oncology, Yonsei Cancer Center, Yonsei University College of Medicine, Seoul, Republic of Korea 